SCIENTIFIC LETTER

\title{
Prevalence and clinical implications of atrial fibrillation episodes detected by pacemaker in patients with sick sinus syndrome
}

\author{
H F Tse, C P Lau
}

Heart 2005;91:362-364. doi: 10.1136/hrt.2003.027219

$\mathrm{R}$ ecent prospective clinical trials have demonstrated that physiologic pacing mode reduces the risk of atrial fibrillation (AF) compared to single chamber ventricular pacing in patients with sick sinus syndrome (SSS). ${ }^{1-3}$ Previous studies have shown that patients with a prior history of AF before pacemaker implant are associated with an increase risk of persistent $\mathrm{AF}^{3}$ Recent advances in the ability to store diagnostic information in implanted devices have provided an opportunity for detection and documentation of AF. The aim of this study was to define the prevalence and clinical significance of device detected AF episodes in patients implanted with pacemakers for SSS.

\section{METHODS}

We studied the clinical characteristics and long term clinical outcome in patients with SSS who had received a dual chamber rate responsive pacemaker (DDDR) for symptomatic bradycardia in our institutions from 1992 to 1998. Patients were eligible if they received a DDDR pacemaker which was capable of providing diagnostic counters for supraventricular tachyarrhythmias and was programmed "ON". P wave analysis was manually measured by a single investigator. A surface echocardiogram was performed in all patients before implant to assess left atrial size, left ventricular ejection fraction, and left ventricular mass.

Data were retrospectively collected regarding demographic characteristics, device diagnostics, clinical status, and outcome during the follow up period. Clinical data were retrieved from the medical records and subsequently during the most recent clinic visit. The following clinical events of each patient were retrieved from their clinical records: the time to the first occurrence of AF; the development of persistent AF; and major cardiovascular events: non-fatal stroke, hospitalisation for congestive heart failure, or cardiovascular death. The AF episode was categorised as either paroxysmal if sinus rhythm was recorded at the subsequent follow up after an episode of AF, or persistent if it was recorded at two consecutive follow up visits.

Continuous variables are expressed as mean (l SD). Statistical comparisons were performed with Student's $t$ test or Fisher's exact test, as appropriate. Cox regression analysis was used to examine the clinical predictors of major cardiovascular events and persistent AF. Actuarial curves for the incidence of persistent AF and major cardiovascular events in patients with and without device detected AF episodes were calculated by the Kaplan-Meier method. A probability value of $\mathrm{p}<0.05$ was considered significant.

\section{RESULTS}

The clinical characteristics of 226 patients included in this analysis are shown in table 1. After a mean follow up of 84 (16) months, 99 patients (44\%) had at least one AF episode documented by the pacemaker. In 30/139 (22\%) patients without a prior history of $\mathrm{AF}$, their pacemaker diagnostic counters detected AF after implant. In 18/87 (21\%) patients with a prior history of AF, their pacemaker diagnostic counters did not detect any AF after implant. Patients with device detected AF episodes were more likely to have a history of AF before pacemaker implant, underlying structural heart diseases, antiarrhythmic drug treatment and anticoagulation use, larger left atria, lower left ventricular ejection fraction and higher prevalence of left ventricular hypertrophy on echocardiography, and longer $\mathrm{P}$ wave duration than patients without AF (table 1).

Persistent AF developed in 49/226 (22\%) patients. Cox regression analysis demonstrated that a prior history of AF before pacemaker implantation (hazard ratio (HR) 4.2, 95\% confidence interval (CI) 2.1 to 10.8; p $<0.001$ ) and the presence of device detected AF episodes (HR 3.1, 95\% CI 1.9 to $12.8 ; \mathrm{p}=0.02$ ) were independent predictors of the development of persistent AF. Kaplan-Meier analysis revealed a significantly higher proportion of persistent AF in patients with a prior history of AF, any device detected AF episodes, or newly device detected AF episodes compared to patients without any AF episodes $(\mathrm{p}<0.001)$ (fig 1 ).

Patients with device detected AF episodes had significantly higher prevalence of major cardiovascular events and nonfatal strokes than those without AF episodes $(\mathrm{p}<0.001)$ (table 1$)$. Cox regression analysis showed that a prior history of AF before pacemaker implantation (HR 2.4, $95 \%$ CI 1.2 to $13.8 ; \mathrm{p}=0.013)$ and the presence of device detected AF episodes (HR 2.0, 95\% CI 1.1 to 15.8; p = 0.020) were independent predictors of major cardiovascular events. Kaplan-Meier analysis revealed a significantly higher prevalence of major cardiovascular events in patients with a prior history of AF, any device detected AF episodes, or new device detected AF episodes compared to patients without any AF episodes ( $p<0.05$ ) (fig 1 ).

\section{DISCUSSION}

Previous clinical studies have reported that up to two thirds of patients with a pacemaker have atrial arrhythmias detected by the devices. ${ }^{4}$ However, the clinical significance of this unexpectedly high incidence of non-sustained device detected atrial arrhythmias remains unclear. In this study, we detected AF episodes in $44 \%$ of our patients during long term follow up, and it was associated with a 10 fold increase in the incidence of persistent AF, and a 2.5-fold increase in major cardiovascular events, especially stroke. Our results confirm the finding of a recent study, ${ }^{5}$ and reveal that the presence of device detected AF episodes, irrespective of symptoms, was an independent predictor for persistent AF and major cardiovascular events. Most importantly, our data

Abbreviations: AF, atrial fibrillation; $\mathrm{Cl}$, confidence interval; $\mathrm{HR}$, hazard ratio; SSS, sick sinus syndrome 
Table 1 Clinical characteristics and outcome of patients with or without device detected atrial fibrillation (AF) episodes by pacemaker diagnostic counters after implantation

\begin{tabular}{|c|c|c|c|}
\hline & $\begin{array}{l}A F \\
(n=99)\end{array}$ & $\begin{array}{l}\text { No AF } \\
(n=127)\end{array}$ & P values* \\
\hline Age (years) & $72(10)$ & $70(10)$ & 0.21 \\
\hline Female (\%) & 56 & 66 & 0.17 \\
\hline Duration of follow up (months) & $88(32)$ & $82(27)$ & 0.19 \\
\hline Prior history of AF (\%) & 55 & 25 & $<0.001$ \\
\hline \multicolumn{4}{|l|}{ Antiarrhythmic } \\
\hline Baseline (\%) & 32 & 5 & $<0.001$ \\
\hline Follow up (\%) & 56 & 6 & $<0.001$ \\
\hline \multicolumn{4}{|l|}{ Anticoagulation } \\
\hline Baseline (\%) & 28 & 3 & $<0.001$ \\
\hline Follow up (\%) & 49 & 6 & $<0.001$ \\
\hline Underlying heart disease (\%) & 76 & 54 & $<0.001$ \\
\hline Hypertension & 37 & 36 & 0.22 \\
\hline Ischaemic heart disease & 36 & 28 & 0.02 \\
\hline Hypertrophic cardiomyopathy & 9 & 13 & 0.42 \\
\hline Thyroid heart disease & 5 & 5 & 0.66 \\
\hline Dilated cardiomyopathy & 2 & 1 & 0.33 \\
\hline Left atrial diameter $(\mathrm{cm})$ & $4.0(0.7)$ & $3.6(0.7)$ & 0.02 \\
\hline Left ventricular ejection fraction & $0.50(0.13)$ & $0.56(0.15)$ & 0.04 \\
\hline Left ventricular mass $(\mathrm{g})$ & 274 (105) & $240(120)$ & 0.21 \\
\hline Left ventricular hypertrophy (\%) & 39 & 18 & $<0.001$ \\
\hline $\mathrm{P}$ wave duration (ms) & $130(21)$ & $122(17)$ & 0.049 \\
\hline Combined cardiovascular events (\%) & 42 & 15 & $<0.001$ \\
\hline Non-fatal stroke (\%) & 32 & 8 & $<0.001$ \\
\hline Hospitalisation for congestive heart failure (\%) & 15 & 10 & 0.31 \\
\hline Cardiovascular death (\%) & 9 & 4 & 0.16 \\
\hline
\end{tabular}

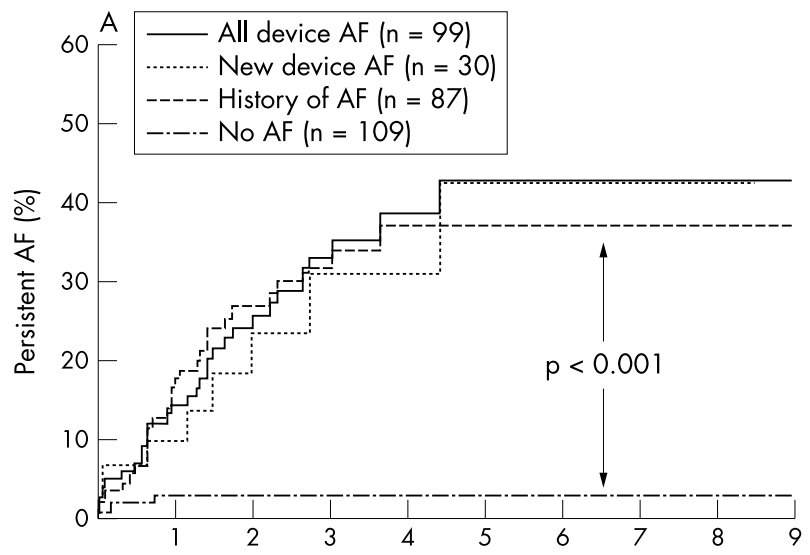

demonstrated the presence of new device detected AF episodes in $22 \%$ patients without a prior history of AF. The occurrence of AF in those patients was also associated with a higher incidence of developing persistent $\mathrm{AF}$ and major cardiovascular events during long term follow up. The use of device detected AF episodes defines a subgroup of patients who did not have a prior history of $\mathrm{AF}$ at higher risk of developing persistent AF and major cardiovascular events. Therefore, it is clinically valuable to detect AF episodes in those patients so that anticoagulation treatment can be initiated to prevent stroke.

In conclusion, in patients with SSS, AF frequently develops after DDDR pacemaker implantation, and progresses to persistent AF over long term follow up. These data suggest the use of a pacemaker diagnostic counter to detect AF episodes in pacemaker patients, especially in those without a prior history of AF, can assist in identifying patients at risk of developing persistent AF and major cardiovascular events, especially stroke. Anticoagulation treatment for stroke prevention should be considered in those patients who have a prior history of AF or device detected AF episodes.

\section{Authors' affiliations}

H F Tse, C P Lau, Cardiology Division, Department of Medicine, Queen Mary Hospital, The University of Hong Kong, Hong Kong

This study was not supported by any funding and no author has a real or perceived conflict of interest.

Correspondence to: Dr Hung-Fat Tse, Cardiology Division, Department of Medicine, The University of Hong Kong, Queen Mary Hospital, Hong Kong, China; hftse@hkucc.hku.hk

Accepted 13 January 2004

\section{REFERENCES}

Figure 1 Kaplan-Meier plots depicting the percentage of patients with (A) persistent $A F$ and $(B)$ major cardiovascular events during long term follow up in patients with a prior history of AF (History of AF), any device detected AF episodes (All device AF), newly device detected AF episodes without a prior history of $A F$ (New device AF), and patients without any history or device detected AF episodes (No AF).

1 Andersen HR, Thuesen L, Baggar JP, et al. Prospective randomized trial of atrial versus ventricular pacing in sick-sinus syndrome. Lancet 1994;344: 1523-8.

2 Lamas GA, Lee KL, Sweeney MO, et al. Ventricular pacing or dual-chamber pacing for sinus-node dysfunction. N Engl J Med 2002;346:1854-62. 
3 Skanes $A C$, Krahn AD, Yee $R$, et al. Progression to chronic atrial fibrillation after pacing: the Canadian trial of physiologic pacing. CTOPP investigators. J Am Coll Cardiol 2001;38:167-72

4 DeFaye $\mathbf{P}$, Dournaux F, Mouton E. Prevalence of supraventricular arrhythmias from the automated analysis of data stored in the DDD pacemakers of 617 patients: the AIDA study. Pacing Clin Electrophysiol 1998;21:250-5.

5 Glotzer TV, Hellkamp AS, Zimmerman J, et al. Atrial high rate episodes detected by pacemaker diagnostics predict death and stroke: report of the atrial diagnostics ancillary study of the mode selection trial (MOST).

Circulation 2003;107:1614-9.

\section{IMAGES IN CARDIOLOGY}

\section{Extracardiac cause of right ventricular outflow tract obstruction}

A 49 year old man was referred to the cardiology clinic for a loud systolic murmur. He was an exsmoker and had a past history of thyrotoxicosis for which he had radioactive iodine treatment followed by current oral thyroxine replacement. His co-morbid problems included hypertension and hyperlipidaemia. He complained of having retrosternal chest discomfort for two years with a recent onset of exertional dyspnoea over two months. In addition, he also had loss of appetite as well as weight loss over the past one year. On examination, he was clinically euthyroid. There was a loud and harsh ejection systolic murmur loudest over the left lower sternal edge with no radiation of the murmur.

A two dimensional echocardiogram was performed. This revealed a large mass lesion adjacent to the right ventricle (panel A: Ao, aorta; LA, left atrium; LV, left ventricle; RV, right ventricle). Colour flow imaging demonstrated a mosaic pattern consistent with that of a turbulent flow across the right ventricular outflow tract (panels $\mathrm{B}$ and $\mathrm{C}$ : AVO, aortic valve orifice; PV, pulmonary valve; RVOT, right ventricular outflow tract). The peak Doppler gradient across the right ventricular outflow tract was $24 \mathrm{~mm} \mathrm{Hg}$ (panel D).

A computed tomogram (CT) of the thorax was performed on the same day. This showed a heterogenous anterior mediastinal mass measuring approximately $8 \times 6 \mathrm{~cm}$ which was invading the pericardium inferiorly (panels E and F). The mass was distorting the superior portion of the right ventricle. It was also displacing the pulmonary trunk posteriorly. Several lobulated hypodensities were seen in the right hepatic lobe and the caudate lobe which were suggestive of hepatic metastases. The patient then underwent a CT guided biopsy of this anterior mediastinal mass.
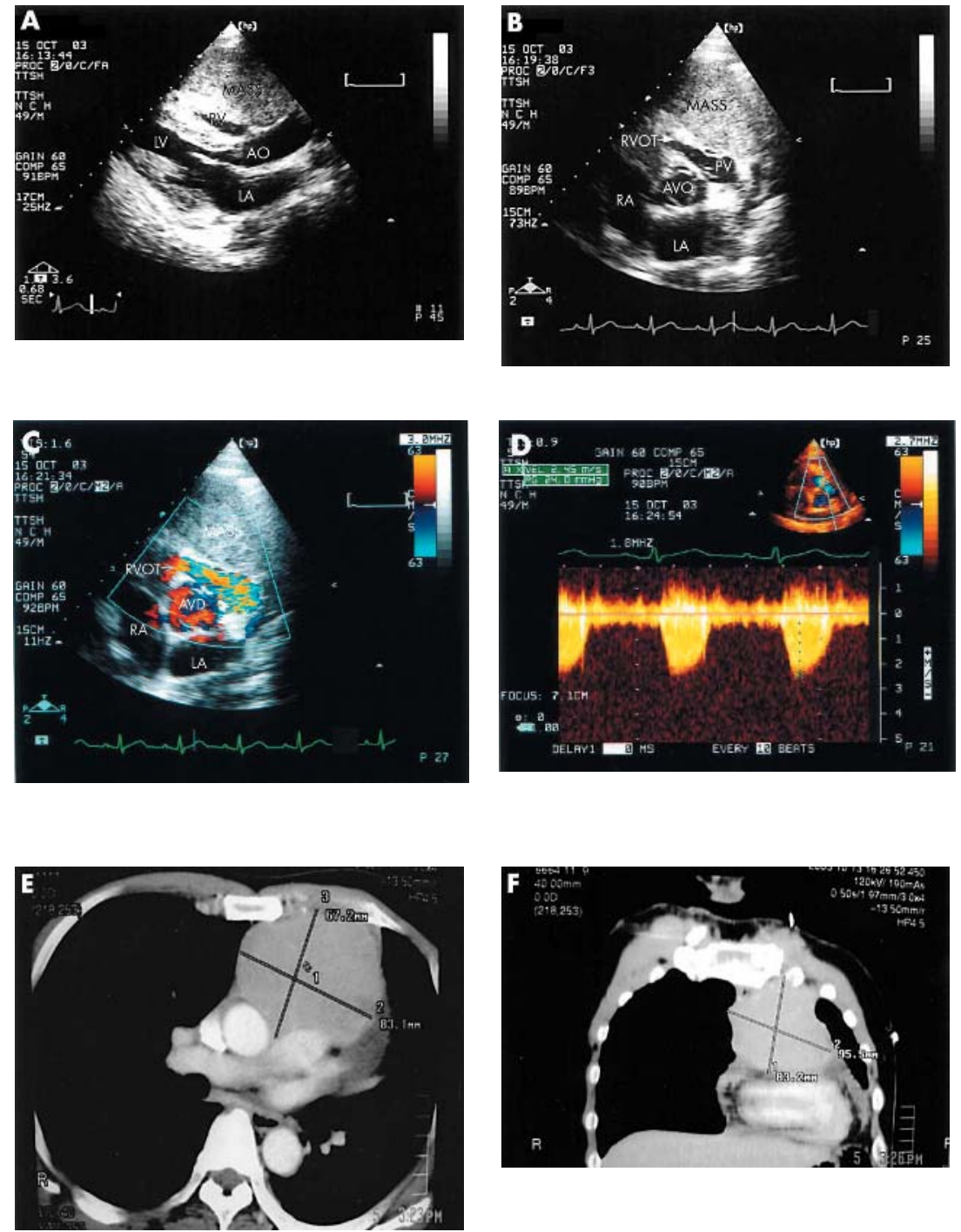

The histopathology of this mass was consistent with that of a thymic carcinoma. He was referred to the oncologist and chemotherapy was planned as the modality of treatment for his condition.

D Foo

$\mathrm{K} \mathrm{Ng}$

david_foo@ttsh.com.sg 